\title{
Serine protease inhibitor Spi2 mediated apoptosis of olfactory neurons
}

\author{
V Thiemmara ${ }^{1,2}$, L Pays ${ }^{1}$, E Danty ${ }^{1}$, F Jourdan ${ }^{2}$, E Moyse ${ }^{1,2}$ \\ and $P$ Mehlen ${ }^{\star, 1}$ \\ ${ }^{1}$ Laboratoire Apoptose et Différenciation-Centre de Génétique Moléculaire et \\ Cellulaire, CNRS UMR 5534, France \\ 2 Neurosciences et Systèmes sensoriels, CNRS UMR 5020, Université Claude \\ Bernard-Lyon1, F-69366 Villeurbanne, France. \\ * Corresponding author: P Mehlen, Apoptosis/Differentiation Laboratory- \\ Molecular and Cellular Genetic Center, CNRS UMR 5534, University of Lyon, \\ 69622 Villeurbanne, France; Tel: (33) 47244 81 90; Fax (33) 4724405 55; \\ E-mail mehlen@univ-lyon1.fr
}

Received 1.2.02; revised 20.5.02; accepted 11.7.02

Edited by G Melino

\begin{abstract}
The olfactory epithelium of adult mouse, where primary sensory neurons are massively committed to apoptosis by removal of their synaptic target, was used as a model to determine in vivo mechanisms for neuronal cell death induction. A macro-array assay revealed that the death of olfactory neurons is accompanied with over-expression of the serine protease inhibitor Spi2. This over-expression is associated with decreased serine protease activity in the olfactory mucosa. Moreover, in vitro or in vivo inhibition of serine proteases induced apoptotic death of olfactory neuronal cells. Interestingly, Spi2 over-expression is not occurring in olfactory neurons but in cells of the lamina propria, suggesting that Spi2 may act extracellularly as a cell death inducer. In that sense, we present evidence that in vitro Spi2 overexpression generates a secreted signal for olfactory neuron death. Hence, taken together these results documenta possible novel mechanism for apoptosis induction that might occur in response to neurodegenerative insults.

Cell Death and Differentiation (2002) 9, 1343-1351. doi:10.1038/ sj.cdd. 4401098
\end{abstract}

Keywords: Serine protease inhibitor; cell death; olfactory neurons; paracrine; axotomy

Abbreviations: NGF, nerve growth factor; GDNF, glial derived neurotrophic factor; TPCK, $\mathrm{N}$ - $\alpha$-tosyl-L-phenylalanine chloromethyl ketone; TLCK, N- $\alpha$ tosyl-L-lysine chloromethyl ketone); GFAP, glial fibrillary acidic protein; DCC, deleted in colorectal cancer)

\section{Introduction}

Cell death is generally accepted as a central mechanism controlling nervous system development ${ }^{1}$ and has also been involved in numerous neurodegenerative diseases. ${ }^{1}$ However, molecular mechanisms that are currently accepted to initiate and drive neural cell death have been mainly reported from non-neural systems or from immortalized neuronal cells. Moreover, the nature of the signals leading to neuronal cell death is mainly unknown. If neurotrophic factors like nerve growth factor (NGF) or glial derived neurotrophic factor (GDNF) have been shown to play an important role for cell survival signalling, ${ }^{1}$ very little information regarding cell death inducers in the nervous system are known. Therefore, the extensive analysis of in vivo systems allowing the study of neural cell death per se is required.

The olfactory epithelium of adult mammals recently emerged as a pertinent model-system for studying the in vivo mechanisms for neuronal apoptosis. ${ }^{2-5}$ This nasal tissue is made up predominantly of chemosensory neurons that are much more liable to apoptosis than most mature neurons of the adult nervous system. Olfactory neurons are known to have a limited lifespan (3-4 weeks) and die through apoptosis spontaneously. 2,3,6 These neurons are renewed by permanent neurogenesis from local neuronal progenitors. $^{7}$ Surgical ablation of their unique target, the olfactory bulb (Figure 1), was shown to trigger massive and synchronous apoptotic death of olfactory neurons, culminating $36 \mathrm{~h}$ after lesion. ${ }^{2,3,8,9}$ This experimental model allowed the analysis of molecular events of apoptosis $^{2,4,5,10}$ from crude tissue extracts of the whole olfactory organ.

Here, mouse olfactory bulbectomy has been used as an in vivo model to investigate the modulation of gene expression associated with neural cell death. While the transcriptional level of the majority of 637 genes studied was not significantly modulated during apoptosis induction of olfactory neurons, the expression of the gene encoding the serine protease inhibitor Spi2 was drastically increased. The serine protease inhibitor Spi2 belongs to the serpin family. This vast family, which shares structural similarity, is supposed to inhibit chymotrypsin-like serine proteinases with an unusual mechanism involving a large conformational change known as the stressed -> relaxed transition. ${ }^{11,12}$ However despite the large number of serpins, very few serpin/serine protease pairs have been identified and only a restricted number of serpins have been shown to inhibit serine protease activity. Numerous roles have been proposed for serpins such as functions in the coagulation cascade, in the control of fibrinolysis or of complement cascade, in the prevention of tissue destruction or metastasis. ${ }^{12,13}$ Recently, several serpins have been reported to be involved in the regulation of cell death (for a review see ${ }^{14}$ ). These numerous roles reflect the diversity of substrates but also a variety of cellular localizations. Some serpins are intracellular proteins and act on intracellular proteinases while others are secreted and affect extracellular proteinases activity (for a review see ${ }^{11}$ ). However, very little information has been reported on the serpin Spi2. Using Spi2 transfection in a neuronal olfactory 
A
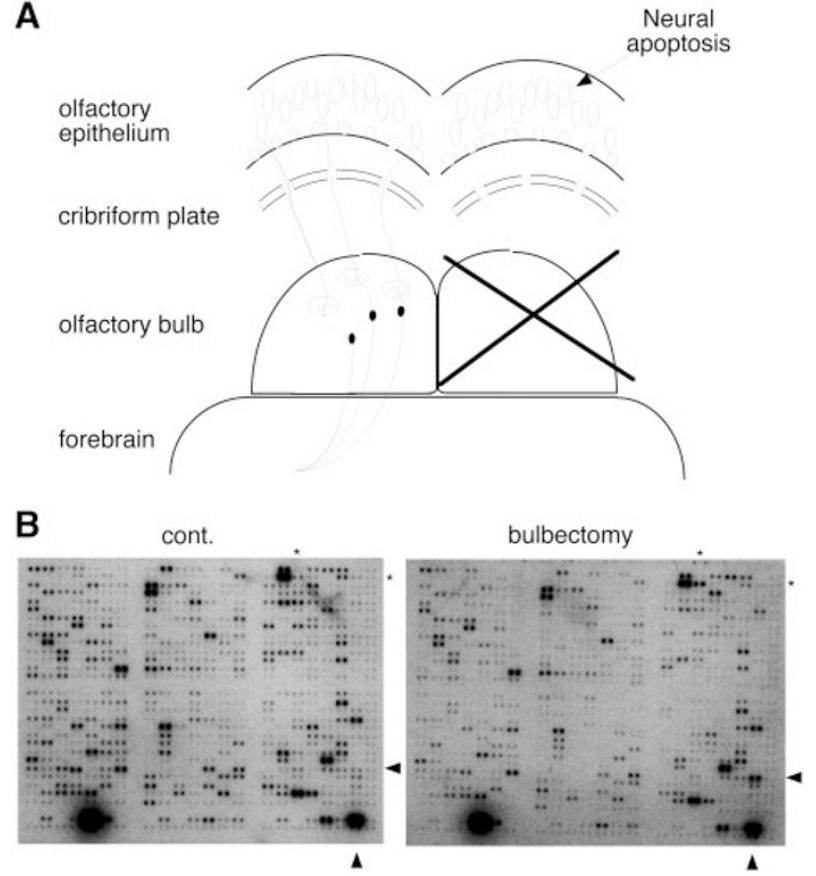

C

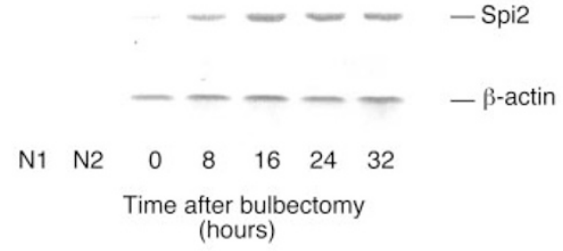

D

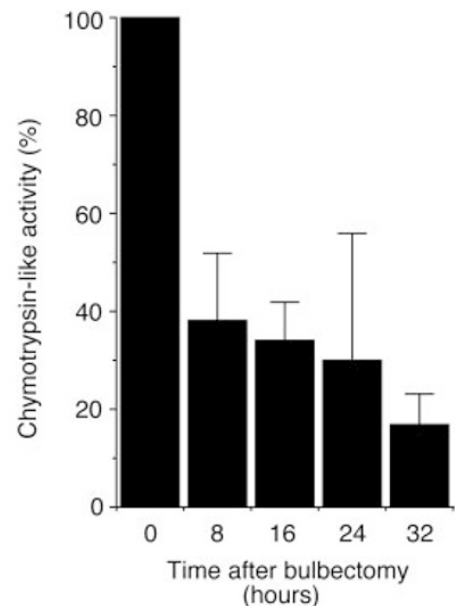

Figure 1 Induction of Spi2 expression during bulbectomy-induced neuronal death in the olfactory epithelium. (A) Diagram of the model system. Removal of the olfactory bulb leads to neural apoptosis of the olfactory epithelium. (B) Olfactory mucosa from either sham (cont.) mice or $18 \mathrm{~h}$ bulbectomized (bulbectomy) mice were removed and RNA were extracted as described in Materials and Methods. Macro-arrays using the Atlas technique were performed as also described in Materials and Methods. Autoradiograms are presented. Arrows indicate the positions of the Spi2 spots. Asterisks indicate the positions of clusterin spots. (C) Expression of Spi2 is associated with neuronal death in the olfactory epithelium. Olfactory mucosa from either 0 (sham), $8,16,24$ or $32 \mathrm{~h}$ bulbectomized mice were removed and then analysed for Spi2 expression by RT-PCR as described in Materials and Methods. $\beta$-actin was used as control. Negative controls: N1; PCR without reverse transcriptase, N2; PCR without cDNA. (D) Bulbectomy induced neuronal death in the olfactory epithelium is correlated with inhibition of a chymotrypsin-like activity. Olfactory mucosa from sham (0) or $8,16,24,32 \mathrm{~h}$ bulbectomized mice were removed, lyzed and further analysed for chymotrypsin-like activity using a Suc-Ala-Ala-Pro-Phe-AMC fluorescent substrate as described in the experimental procedures. An index of chymotrypsin-like activity is presented as the ratio between the different bulbectomized samples and the control olfactory mucosa lysate sample. Note the massive decrease of chymotrypsin-like activity as soon as $8 \mathrm{~h}$ after bulbectomy

cell line and treatment with a permeant serine protease inhibitor, we demonstrated both in vitro and in vivo that serine protease inhibition leads to neural cell death, hence suggesting the involvement of serine protease activity in the regulation of neural apoptosis. Of interest, the cell death-inducing activity of Spi2 seems to act via a paracrine system since we report here that the expression of Spi2 (i) occurs outside the olfactory epithelium, in the underlying lamina propria and (ii) results in the release of an extracellular signal that drives in vitro death of olfactory neurons.

\section{Results}

\section{Spi2 expression is induced in the olfactory mucosa concomitantly with the death induction of olfactory neurons}

The massive and synchronous apoptosis observed in olfactory epithelium after olfactory bulb ablation was used as a model to monitor gene expression pattern during neural apoptosis induction. Macro-arrays containing 637 probes were hybridized with reversed RNA from the whole olfactory mucosa (epithelium + underlying connective tissue and olfactory nerve bundles) of either mock or $18 \mathrm{~h}$ bulbectomized mice. The time of $18 \mathrm{~h}$ after bulbectomy was picked because at this early step apoptosis was just started, with yet no morphological changes of the epithelium. ${ }^{2}$ Figure 1B and Table 1 show that in agreement with a previous observation, clusterin expression is modulated in response to bulb withdrawal ${ }^{10}$ supporting the array as an adequate tool for gene expression analysis in the olfactory system. Expression of some apoptosis-related genes appeared also to be either induced (e.g., Akt kinase, p65 NF- $\kappa \mathrm{B}, \mathrm{Bcl}-\mathrm{XI}, \mathrm{RIP}$ ) or inhibited (e.g., caspase-7, BAG1). However, in each case these changes were moderate. Moreover, most of the genes spotted in the array and mainly all the typical apoptosis-related genes did not show any important change in their levels of expression. One exception was visualized with the gene encoding for the serine protease inhibitor Spi2 that showed a massive overexpression, i.e., more than 20 -fold at onset of neuronal apoptosis. 
Table 1 Genes activated or repressed 18 hours after bulbectomy

\begin{tabular}{lcll}
\hline Genes induced after bulbectomy: & Ratio & Genes repressed after bulbectomy: & Ratio \\
\hline c-Akt proto-oncogene & 3.03 & AT motif-binding factor ATBF1 & 0.07 \\
cathepsin H & 3.06 & HMG-box transcription factor & 0.08 \\
NFkB transcription factor p65 subunit & 3.09 & Caspase-7 & 0.10 \\
Ubiquitin-conjugating enzyme yeast Rad6 homolog & 3.19 & Vav & 0.12 \\
PCNA & 3.40 & Transcription factor UBF & 0.12 \\
MHR23B & 3.43 & Bone morphogenic protein 1 & 0.12 \\
Nm23-M2 & 3.56 & Collagenase type IV & 0.17 \\
TDAG51 & 3.88 & Heat shock transcription factor 2 & 0.18 \\
Bcl-XI & 4.22 & Cyclin D3 & 0.21 \\
RIP cell death protein & 4.24 & Gelatinase B & 0.21 \\
DNAse I & 4.94 & ATP-binding cassette 8 0.22 & 0.22 \\
CD14 antigen & 6.63 & Macrophage mannose receptor & 0.23 \\
Clusterin & 10.36 & Fra-2 & 0.26 \\
Pur-alpha transcriptional activator & 14.79 & Glutamate receptor NMDA 2B & 0.28 \\
Spi2 & 27.3 & Homeo box protein 4.2 & 0.29 \\
& & BAG-1 & 0.29 \\
& & NF2 & \\
& & Ets-2 transcription factor & 0.31 \\
\end{tabular}

From the 627 genes listed in the Atlas mouse cDNA expression array (see www.clontech.com/archive/JAN98UPD/Atlaslist.htlm for the complete list), the genes either induced or repressed $18 \mathrm{~h}$ after bulbectomy are presented. For each gene, the value in brackets indicates the ratio between the value obtained in the bulbectomy experiment and the value of the control experiment. Values above threefold repression or activation are shown

\section{Spi2 expression during neuronal death in the olfactory epithelium is associated with a decrease of serine protease activity}

The serine protease inhibitor Spi2 belongs to the serpin family and this protein displays an anti-chymotrypsin activity. ${ }^{15}$ To analyse further the role of serpins in olfactory neural cell death, we first analyzed whether the detected over-expression of Spi2 in olfactory mucosa during apoptosis induction was associated with a decrease in serine protease activity. While Spi2 mRNA rapidly accumulates in olfactory mucosa after bulbectomy as monitored by both RT - PCR (Figure 1C) or Northern blot (not shown), we observed a significant decrease (up to fivefold) of serine protease activity, measured by the ability of olfactory mucosa lysate to catalyse the cleavage of a chymotrypsin fluorescent substrate (Figure 1D). It is then interesting to note that the accumulation of Spi2 mRNA and the decrease of serine protease activity were detected concomitantly less than $8 \mathrm{~h}$ after bulbectomy. Hence, during the initiation step of neuronal apoptosis in the olfactory epithelium, Spi2 over-expression is associated with the inhibition of serine protease activity.

\section{Serine protease inhibitors induce neural cell death}

We then checked the role of Spi2 expression in cell death control. Spi2 expressing construct was transiently transfected in 13.S.1.24 cell line, a cell line obtained from the immortalization of rat olfactory neuroblasts. ${ }^{16}$ Transient transfection in this neuronal cell line generally allows $30-$ $40 \%$ of transfected cells as measured by a control transfection with a $\beta$-gal expressing vector (not shown and ${ }^{17}$ ). Cell death was monitored by Trypan blue exclusion staining $48 \mathrm{~h}$ after transfection. ${ }^{18}$ Of interest, expression of Spi2 led to a four to five times increased 13.S.1.24 cell death compared to control transfected cells (Figure 2A). Spi2-induced cell death was defined as apoptosis since the increased Trypan blue staining was accompanied with DNA fragmentation (Figure 2B) and caspase activation (Figure $2 \mathrm{C}$ ). Hence, serine protease inhibitor Spi2 expression, a phenomenon occurring in vivo during olfactory neural cell death, induced apoptosis of immortalized neural cells. Since many serpins have shown functional roles that are independent of their serine proteinase inhibitory activities (for a review see ${ }^{11}$ ), we checked whether Spi2 was specifically required, or if the pro-apoptotic activity was more generally related to serine protease activity inhibition. 13.S.1.24 cells were then incubated with the antichymotrypsin compound $\mathrm{N}$ - $\alpha$-tosyl-L-Phenylalanine chloromethyl ketone (TPCK). ${ }^{19}$ As shown in Figure $3 \mathrm{~A}, \mathrm{~B}, \mathrm{C}$, a $5 \mathrm{~h}$ incubation with TPCK led to an important apoptosis induction of 13.S.1.24 cells monitored by caspase activation (Figure $3 B$ ), while it concomitantly drove serine protease inhibition (Figure 3A). As a control experiment, treatment with $\mathrm{N}-\alpha$ tosylL-lysine chloromethyl ketone (TLCK), a specific trypsin-like serine protease inhibitor, had no effect on cell viability or caspase activity (not shown). Hence, inhibition of serine protease activity and more specifically of chymotrypsin-like activity may represent a pro-apoptotic stimulus.

\section{In vivo inhibition of serine protease activity induces apoptosis in the olfactory epithelium}

To analyse whether Spi2 expression and serine protease inhibition occurring during apoptosis induction in the olfactory mucosa represent initiating steps for cell death, the olfactory epithelium was treated in vivo with the membrane-permeant anti-chymotrypsin TPCK. Remarkably, $18 \mathrm{~h}$ after TPCK treatment apoptosis induction monitored by TUNEL reactivity was observed in the intermediate sublayer of olfactory epithelium while in solvent injected control animals, the olfactory epithelium remained intact (Figure 3D). The frequency and spatial patterning of TPCK-induced TUNEL 
A

B
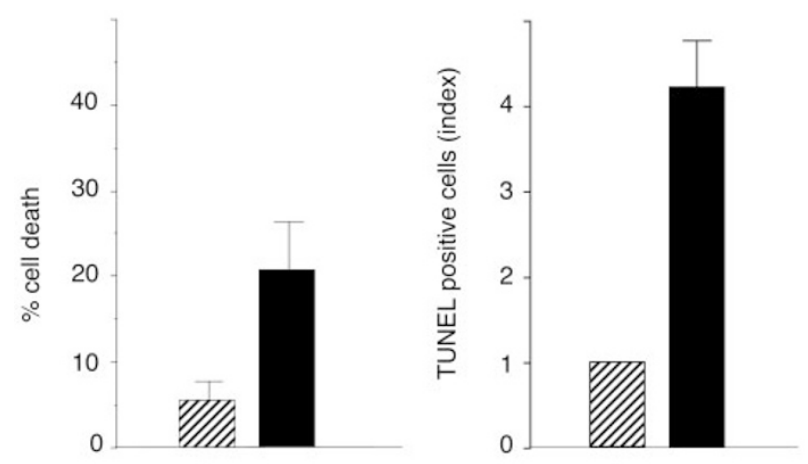

C

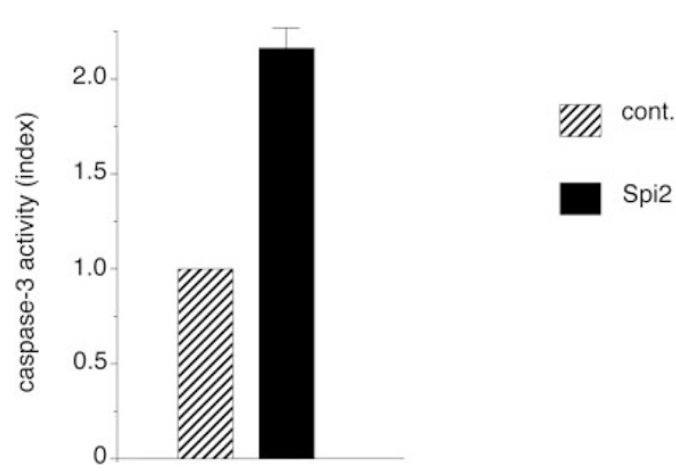

Figure 2 Spi2 expression induces neural apoptosis. 13.S.1.24 cells were transiently transfected with mock plasmid pcDNA3.1 (cont.) or the Spi2 expressing plasmid pcDNA3-Spi2. (A) $48 \mathrm{~h}$ after transfection, cell death was measured by the Trypan blue exclusion assay. Percentage of cell death is presented as the percentage of Trypan blue positive cells. Standard deviations are indicated $(n=3)$. (B) TUNEL labelling was performed as a marker for DNA fragmentation as described in Materials and Methods. An index of TUNEL positive cells is presented as the ratio between the percentages of TUNEL positive cells in Spi2 transfected cells versus control-transfected cells. (C) Caspase activity was measured using the DEVD-fluorescent substrate as described in Materials and Methods. Caspase activation is presented as the ratio between the caspase activity of the sample and that measured in 13.S.1.24 cells transfected with pcDNA3.1. Standard deviations are indicated $(n=3)$

reactivity indicate that apoptotic cells are mature olfactory neurons. ${ }^{7,10}$ Furthermore, Figure 3D shows that TPCKinduced apoptosis of olfactory neurons led to a decrease of olfactory epithelium thickness very similar to the one following olfactory bulb ablation. ${ }^{10}$ These data strongly support the view of a specific apoptosis inhibitory activity of serine protease in olfactory neurons of the normal olfactory epithelium. This would suggest that in vivo TPCK-induced serine protease inhibition has led to a rapid activation of the cell death machinery as observed in vitro (Figure 3B, C).

\section{Spi2 mRNA over-expression occurs in cells of the lamina propria}

Serine protease inhibitors are known to act either as intracellular proteins or as proteins released in the extracellular environment. ${ }^{11}$ To our knowledge, no report has indicated whether Spi2 was an intracellular protein or a secreted protein. It was then possible to define Spi2 effect on cell death either as a direct intracellular pathway for apoptosis induction or as a paracrine effect. To answer this question, we first sought to determine the localization of Spi2 transcripts within the olfactory mucosa. In situ hybridization experiments were then performed on olfactory organ sections of unilaterally bulbectomized mice. They confirmed that Spi-2 over-expression was induced in the olfactory mucosa since an increase of labelling was observed ipsilaterally to the lesion. However, no specific labelling of Spi2 mRNA was detected in olfactory neurons, the increased labelling being actually strictly restricted to cells located in the underlying lamina propria, around the axon bundles of olfactory nerve (Figure 4A, B).

\section{Spi2 acts as a paracrine inducer for cell death}

The fact that Spi2 expression does not occur within olfactory neurons favoured the hypothesis that Spi2 may act as an extracellular signal for olfactory neuron death induction. Therefore, we sought to determine the cellular localization of Spi2 protein. However, we failed to detect Spi2 protein, even when Spi2 was motif-tagged. In the absence of a possible immuno-detection, we then sought to determine whether Spi2 was able to induce cell death via a paracrine mechanism. 13.S.1.24 cells were then grown with the culture medium of respectively mock or Spi2 transfected cells. As shown in Figure $4 C, D$, apoptosis induction, monitored by the measurement of caspase activity (Figure 4C) or TUNEL staining (Figure 4D), was observed in the cells cultured with the medium of Spi2-transfected cells. A possible bias of this experiment is that cells expressing Spi2 may be dying and then possibly produce and release toxin or cell death stimuli, which in turn would be indirectly the cause of the paracrine induction of cell death by Spi2. To exclude this possibility, we performed a similar experiment with culture medium from cell transfected with deleted in colorectal cancer (DCC), a protein that is known to drive apoptotic cell death. ${ }^{18}$ As shown in Figure $4 \mathrm{C}, \mathrm{D}$, the culture medium of DCC expressing cells has no ability to induce 13.S.1.24 cell death, hence strongly suggesting that Spi2, either directly if secreted or indirectly by cleaving a protein that is then released, induced apoptosis of the 13.S.1.24 olfactory neuronal cell line by a paracrine mechanism.

\section{Discussion}

The present work sought to study neuronal apoptosis mechanisms in vivo by using the model of the mouse olfactory epithelium. $^{2-5}$ A macro-array analysis was set to monitor possible modulations of gene expression during the massive apoptosis observed in the olfactory epithelium after olfactory bulb ablation. The results obtained from the macro-array assay led to several conclusions. First, this assay was validated by the finding that the clusterin mRNA is overexpressed after bulbectomy, a result described a few years ago. ${ }^{10}$ The second conclusion is that, beside expectations, the transcriptional status of the vast majority of the genes (605 genes out of 637) was not significantly modulated in response to bulbectomy. Of interest, the bulbectomy, that induced the 
A

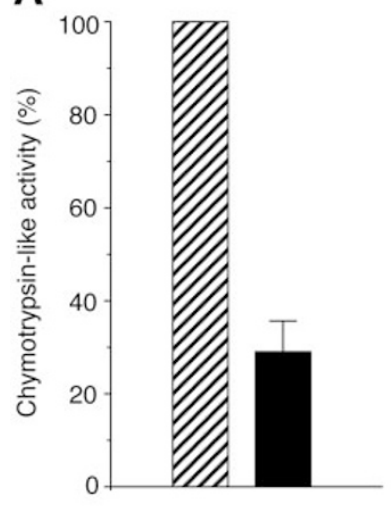

B

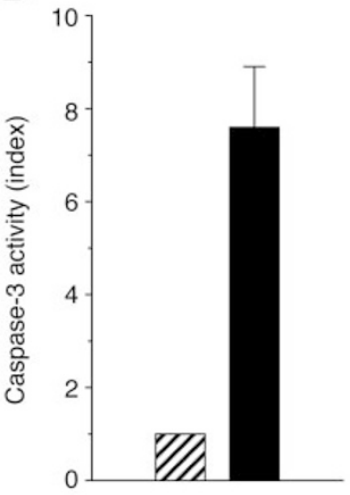

C

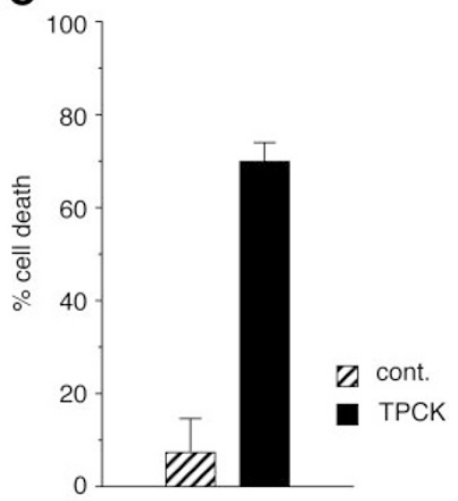

D

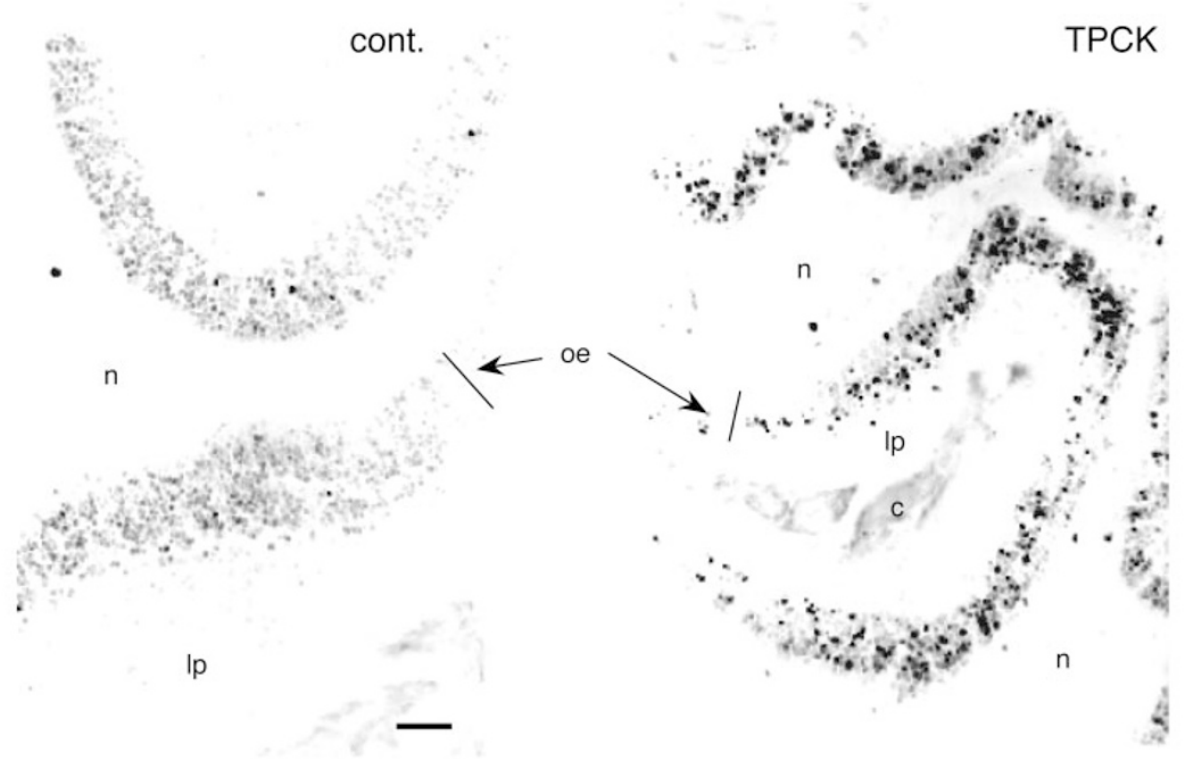

Figure 3 Serine protease inhibition induces neural apoptosis in vitro and in vivo. 13.S.1.24 cells were incubated with or without $25 \mu \mathrm{M}$ TPCK (Sigma) for $5 \mathrm{~h}$ and were then analyzed either for chymotrypsin-like activity (A) as described in Figure 1D or for caspase activation as a measurement of apoptosis (B) or for Trypan blue exclusion as a measurement of cell death (C). Standard deviations are indicated $(n=3)$. (D) TUNEL assay was performed on olfactory epithelium treated for $18 \mathrm{~h}$ with or without TPCK as described in Materials and Methods. Note after TPCK treatment the increased number of TUNEL positive cells and the thinness of the olfactory epithelium. $\mathrm{c}=$ cartilage; Ip=lamina propria (or chorion); $n=$ nasal cavity; oe=olfactory epithelium. Scale bar: $50 \mu \mathrm{m}$

massive and synchronous death of olfactory neurons, did not alter the general expression profile of apoptosis-related genes. Moreover, except the observed over-expression of RIP (by a limited factor of about 4), a gene encoding for a tyrosine kinase protein involved in death receptor signalling, ${ }^{20}$ most of these minor changes in pro-apoptotic or anti-apoptotic genes expression would theoretically result in an antiapoptotic effect (e.g.; down-expression of caspase-7, overexpression of Akt). This observation does not preclude the likely involvement of standard intracellular signals in apoptotic death of olfactory neurons following target deprivation ${ }^{5}$ but strongly suggests the transcriptional-independent nature of these signals. Finally, our current finding of Spi2 overexpression strongly suggests that alternative pathways could also play an important role in olfactory neurons commitment to death.

Here, the macro-array analysis followed by in vitro and in vivo experiments led to propose that the death of olfactory neurons is associated with a serine protease inhibitor (Spi2) over-expression. This over-expression that occurs in the lamina propria but not in the olfactory neurons may then induce an important inhibition of serine protease activity locally within the olfactory epithelium, a phenomenon that drives apoptosis induction of olfactory neurons. These results then define the implication of serine protease inhibition in neuronal cell death in vivo. The involvement of serine proteases and serine protease inhibitors in cell death regulation is, however, not totally a new concept. 


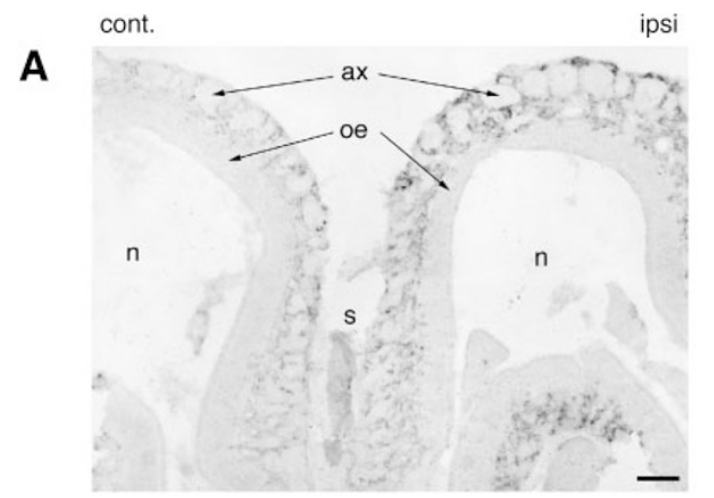

B
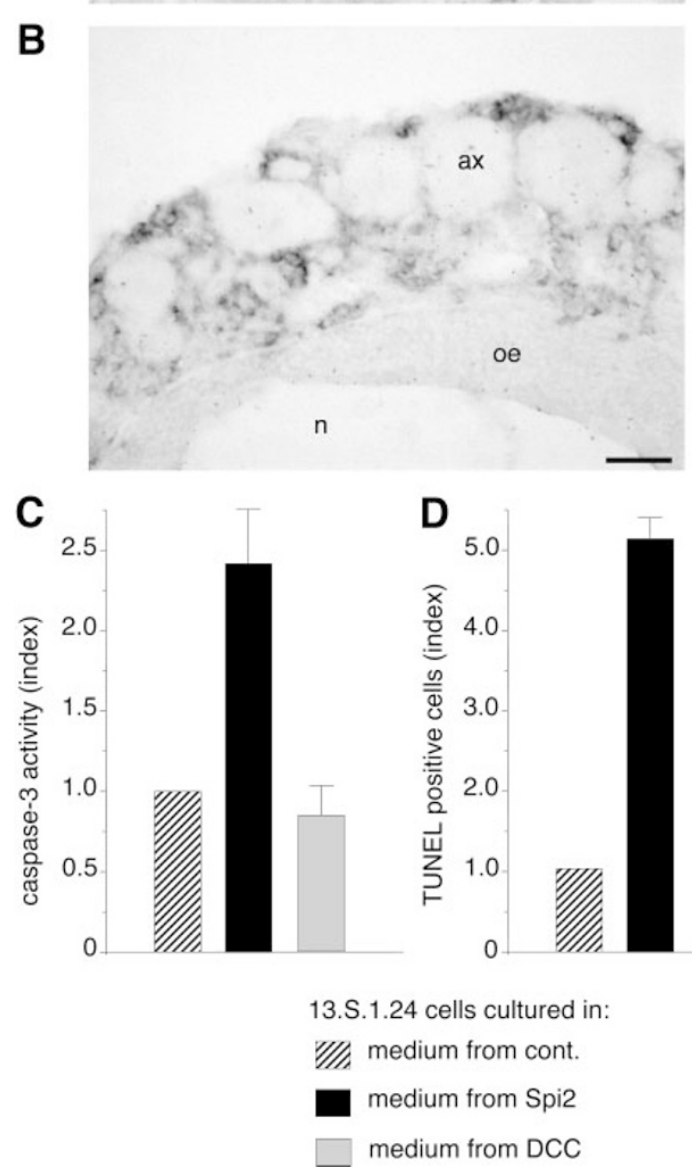

Figure 4 Spi2 is expressed by cells of the olfactory lamina propria and induces cell death of olfactory neurons in a paracrine mechanism. In situ hybridization using Spi2 antisense cRNA probe was performed on olfactory mucosa of $18 \mathrm{~h}$ unilateral bulbectomized mice as described in Materials and Methods. (A) The left part of the figure shows the olfactory mucosa corresponding to the unlesioned side while the right part regards the olfactory mucosa ipsilateral to the lesion. Scale bar $100 \mu \mathrm{m}$. (B) Magnification of an ipsilateral olfactory mucosa. Scale bar $200 \mu \mathrm{m}$. Note that cells located in the lamina propria and surrounding axon bundles are strongly labelled. $\mathrm{n}$ : nasal cavity, s: nasal septum, oe: olfactory epithelium, ax: axon bundle (olfactory nerve), ipsi: bulbectomized side, control: unlesioned side. (C,D) Spi2 may act as a paracrine inducer of cell death. 13.S.1.24 cells were transfected for $72 \mathrm{~h}$ either with a mock pcDNA3, with Spi2 expressing construct pcDNA3-Spi2 or with pCMV-DCC-S ${ }^{18}$. After $24 \mathrm{~h}$ of transfection and then every $8 \mathrm{~h}$, part of the culture media of these transfected populations was used to grow 13.S.1.24 cells. Caspase activity assay (C) or TUNEL staining (D) was then performed on 13.S.1.24 cells cultured with medium from either mock or Spi2-transfected cells as described in Figure 2B,C. Standard deviations are indicated $(n=3)$
Indeed, several other serpins have been recently associated with cell death but surprisingly they seem to inhibit apoptosis. For example the poxvirus Cytokine response modifier $\mathrm{A}(\mathrm{CrmA}),{ }^{14}$ the poxvirus serine protease inhibitor1 (SPI-1), ${ }^{12}$ the proteinase inhibitor 9 (PI-9), ${ }^{14}$ the plasminogen activator inhibitor 2 (PAl-2), ${ }^{12}$ the protease nexin I $(\mathrm{PN}-\mathrm{I})^{21}$ were demonstrated to inhibit apoptosis in vitro. Moreover, all these serpins are believed to modulate cell death by interfering with the caspase-signalling pathway. Hence, CrmA is a classic inhibitor of the 'death receptor'signalling pathway and is known to block caspase- 8 by direct interaction. ${ }^{14}$ Along the same line, the serine protease Granzyme $B$ was shown to drive apoptosis because this serine protease displays aspartase activity and then mimics caspase activity. ${ }^{14}$ Our results showing that Spi2 over-expression in cells of the lamina propria provokes apoptosis in olfactory neurons suggests that serine protease inhibition may also be considered as an extracellular signal for cell death induction. In that sense, we observed that in vivo treatment of the olfactory epithelium with TPCK, an anti-chymotrypsin protease, also induces apoptosis of the olfactory epithelium. This would then suggest that the signal of serine protease inhibition is sufficient to induce the commitment of olfactory neurons to undergo apoptosis.

Interestingly, Spi2 expression was monitored in the lamina propria. The in situ hybridization signal strikingly overlaid the glial sheath of olfactory nerve bundles and resembled the staining of ensheathing cells. ${ }^{22}$ Even though we cannot exclude that other cells from the lamina propria may actually be the Spi2 expressing cells, ensheathing cells would represent good candidates for being the sensor of the axotomy insult. Indeed, these cells surround fascicles of primary olfactory axons en route to the olfactory bulb and appear more and more of crucial importance for olfactory neuronal turn-over. ${ }^{23-25}$ Indeed, ensheathing cells display an unequalled property to promote axonal growth ${ }^{26}$ that was recently used for transplantation/repair strategy following spinal cord injury in adult rat. ${ }^{27}$ These glial cells probably play a crucial role for neurogenesis that occurs throughout life span in the olfactory system. It would then be tempting to propose that olfactory ensheathing cells play also a role in neural death induction by producing and releasing a pro-apoptotic molecule like Spi2. However we have failed to detect Spi2 protein using either specific immunosera or motif-tagged Spi2. Thus, the fact that Spi2 is an extracellular protein is mainly speculative. However, sequence comparison with other known secreted serine protease inhibitors have revealed the presence of a putative extracellular targeting signal of 20 amino acids in the N-terminus of the Spi2 protein (prediction obtained from SignalP V1.1 World Wide Web Server). If it holds true, this would support the role of Spi2 as a paracrine signal for cell death induction.

A tempting view would then be that extracellular Spi2 presence in the environment of olfactory neurons inhibits specific serine protease(s) expressed by these neurons but probably located in their immediate extracellular matrix. We then have to consider that, in the absence of Spi2, this (these) protease(s) display(s) a constitutive survival activity 
for olfactory neurons. However, understanding this survival activity passes through the determination of the Spi2 target and remains to be investigated. The present study suggests serine protease inhibition as a pro-apoptotic mechanism that contributes to apoptosis in adult olfactory neurons deprived from their synaptic targets. Most other adult mammal neurons were shown to survive to target withdrawal (i.e. axotomy, target deprivation). Commitment to apoptosis of olfactory neurons therefore implies either defect of anti-apoptotic mechanisms or additional proapoptotic mechanisms that enhance cell liability to selfdestruction. Axotomy-induced Spi2 accumulation in the olfactory epithelium may then account for the specific sensitivity of olfactory neurons to apoptosis. It might also be involved in the unusual short life span of adult olfactory neurons (i.e. $3-4$ weeks). ${ }^{6}$ However, a recent similar macro-array approach demonstrated that in experimental allergic encephalitis mice, ${ }^{28}$ oligodendrocyte death is associated with a similar increase of Spi2 expression (10fold increase) (Nataf S., personal communication). Similarly, treatment with the serine protease inhibitor TPCK was shown recently to enhance post-traumatic brain injury effects, probably by triggering neuronal apoptosis. ${ }^{29}$ Finally, a strain-specific absence of Spi2 expression in mouse hippocampus was associated with an increased resistance to seizure-induced cell losses. ${ }^{30}$ It would then be tempting to postulate that serine protease inhibition might be a rather general mechanism that is associated with degeneration and cell death. It would then be of particular interest to evaluate whether Spi2 is overexpressed in other degenerative pathologies involving apoptosis excess. If this holds true, induction of serine protease activity to prevent or delay degenerative disease and ageing might appear as an attractive therapeutic approach.

\section{Materials and Methods}

\section{Animals and tissues preparation}

Uni- or bilateral bulbectomy on 3-month-old male mice of the C57BI/6J strain was performed under anesthesia (Equithesin $0.3 \mathrm{~g} / 0.3 \mathrm{ml}$ per $100 \mathrm{~g}$ body weight) as follows. One or two symmetrical $1 \mathrm{~mm}$-wide holes were dug with a dental drill in the dorsal face of the skull on each side of the medial suture, $2 \mathrm{~mm}$ rostro-laterally to bregma. A curved glass pipette connected to a vacuum pump was then introduced through each hole and the underlying olfactory bulb was totally removed by aspiration. The resulting space was filled up with gelfoam and the dorsal head skin was sutured. Bulbectomized mice were housed and allowed to recover from anesthesia. Animals were sacrificed by decapitation at adequate post-lesion times; the bulk of olfactory turbinates was immediately dissected out, using RNAse-free tools, and either placed in sterile microtubes for nitrogen liquid freezing and subsequent biochemical assays or vacuum-embedded in histological mounting medium (OCT, Miles, UK) for snap-freezing in isopentane at $-80^{\circ} \mathrm{C}$ and subsequent cryomicrotomy. Each frozen organ was crumbled, immediately dipped into either $1 \mu \mathrm{l}$ TRIzol reagent (Life Technologies) for RNA extraction or $500 \mu \mathrm{l}$ of SDSLaemmli buffer for protein analysis. In vivo treatment of olfactory epithelium with TPCK (Sigma) was performed on anesthetized mice by inoculating each naris with $10 \mu \mathrm{l}$ of $400 \mu \mathrm{M}$ TPCK solution. TPCK solution was prepared in $\mathrm{NaCl} 0.9 \%$ solution by using a stock solution of TPCK $10^{-2} \mathrm{M}$ in DMSO. All mice were allowed to recover and sacrificed by decapitation $18 \mathrm{~h}$ later.

\section{Macro-array assay}

The Atlas cDNA expression arrays system (Clontech) was used according to manufacturer's instructions. Two pools of RNA were prepared from 10 olfactory organs of either $18 \mathrm{~h}$ bulbectomized mice (apoptosis onset) or intact mice (control). These RNA were reverse transcribed using the Moloney Murine Leukemia Virus ReverseTranscriptase (MMVL-RT) $(50 \mathrm{U} / \mu \mathrm{l})$ in the presence of $\alpha-{ }^{32} \mathrm{P}$ dATP (3.5 $\mu \mathrm{Ci} / 5 \mu \mathrm{g} \mathrm{ARN}$ ) as described in the Atlas procedure. The resulting labelled mixtures were applied separately on two identical probebearing membranes which have been pre-saturated with salmon testes DNA-contained hybridization mix (Express Hyb; Clontech), at $68^{\circ} \mathrm{C}$ for $30 \mathrm{~min}$. Hybridization was conducted overnight at $68^{\circ} \mathrm{C}$ and the membranes were washed at $68^{\circ} \mathrm{C}: 4 \times 20$ min washes in SSC $2 X$; SDS $1 \%$, and $2 \times 20$ min washes in SSC $0.1 X$; SDS $0.5 \%$. Membranes were then enclosed in Saran ${ }^{(i)}$ wrap and placed in a PhosphorImager $^{(i x)}$ (BioRad) for $24 \mathrm{~h}$. Plots density was quantified using QuantiOne $^{(i}$ software (BioRad) and normalized by reference to several housekeeping genes. Comparison was made between the signals obtained, for each CDNA, in the two situations: control/ apoptosis onset.

\section{RT-PCR}

Total RNA from olfactory organs of mice sacrificed every $8 \mathrm{~h}$ following bulbectomy were reverse transcribed by incubating for $1 \mathrm{~h}$ at $42^{\circ} \mathrm{C}$ $5 \mu \mathrm{M}$ random hexamers, $1 \mathrm{mM} \mathrm{dNTP}, 10 \mathrm{mM}$ DTT and $200 \mathrm{U}$ of the Superscript reverse transcriptase (Life Technologies). Spi2 specific PCR reactions were performed using 5'-ATGGCTTTCATTGCAGCTCTGGGG-3' and 5'-TCATTTGGGGTTGGCTATCTTGGC-3 as primers. A control PCR was performed on $\beta$-actin with the following primers 5'-ATGGATGACGATATCGCTGCGCTG-3' and 5'-CTAGAAGCACTTGCGGTGCACGAT-3'.

\section{Construct, cell lines, transfection}

The Spi2 construct pEB-II was provided by A LeCam. The coding sequence of Spi2 was inserted in Hindlll-BamH1 cloning sites of pcDNA3.1 (InvitroGen). The HA-Spi2 expressing construct was obtained by inserting in pcDNA3-TOPO, the PCR product generated with the following primers 5'-GGCATCGATATGTATCCATATGATGTTCCAGATTATGCTATGGCCTTC-3' and 5'-GCCATCGATAAGATTTTCCTAATGCCC-3' and pCDNA3-Spi2 as template. The 13.S.1.24 rat olfactory neuroblast cell line $^{16}$ was cultured in Dulbecco's modified medium (Life Technologies) supplemented with $10 \%$ SVF (Life Technologies), $0.4 \%$ fungizone (Life Technologies) and $0.1 \%$ Gentamycin (Life Technologies). Transient transfections were performed as previously described ${ }^{18}$ using Lipofectamine Plus Reagent (Life Technologies).

\section{In situ hybridization}

Sense and antisense digoxigenin (DIG)-labelled RNA probes for Spi2 were generated from pcDNA3 - Spi2 plasmid according to the methods described in the Roche 'DIG RNA labelling kit'. In situ hybridization was performed on coronal cryostat sections through the olfactory mucosa of unilaterally bulbectomized adult mice. Briefly, slides were successively treated with $10 \mu \mathrm{g} \mathrm{ml}$ proteinase $\mathrm{K}$ (Roche) $5 \mathrm{mn}$ at 
$37^{\circ} \mathrm{C}$, and with acetic anhydride in $0.1 \mathrm{M}$ triethanolamine for $10 \mathrm{~min}$. They were then dehydrated through graded ethanol solutions and hybridization was performed at $56^{\circ} \mathrm{C}$ for $15 \mathrm{~h}$. Slides were washed in SSC at $56^{\circ} \mathrm{C}$, followed by a formamide wash at $56^{\circ} \mathrm{C}$ and SSC washes at $37^{\circ} \mathrm{C}$. DIG-labelled RNA hybrids were reacted with alkaline phosphatase-conjugated anti-DIG antibodies (Roche). Reaction product was visualized by incubating the sections with nitro-blue tetrazolium chloride and 5-bromo-4-chloro-3-indolylphosphate (Roche) overnight at room temperature.

\section{Cell death assays}

Cells viability was assessed using Trypan blue exclusion assay as described previously. ${ }^{18}$ Measurement of caspase activity was performed using the ApoAlert Caspase-3 assay (Clontech) according to manufacturer's instruction and as described previously. ${ }^{18}$ In situ labelling of apoptotic cells was performed by the TUNEL procedure (TdT-mediated dUTP nick-end labelling). ${ }^{6,10}$ Frozen sections of olfactory organ were fixed in buffered $4 \%$ paraformaldehyde solution, rinsed and treated $5 \mathrm{~min}$. with proteinase $\mathrm{K}(20 \mathrm{mg} / \mathrm{ml}$ in PBS). Sections were then rinsed and incubated $10 \mathrm{~min}$ with $3 \% \mathrm{H}_{2} \mathrm{O}_{2}$. After 5 min pre-incubation in TdT buffer (Tris- $\mathrm{HCl} 30 \mathrm{mM} \mathrm{pH} 7.5$ containing $140 \mathrm{mM}$ sodium cacodylate and $1 \mathrm{mM}$ cobalt chloride), sections were incubated $1 \mathrm{~h}$ at $37^{\circ} \mathrm{C}$ with $\mathrm{TdT} 300 \mathrm{U} / \mathrm{ml}$ (Roche) and $6 \mu \mathrm{M}$ biotinylated dUTP (Roche) in TdT buffer, then rinsed $15 \mathrm{~min}$ in TB buffer ( $\mathrm{NaCl} 300 \mathrm{mM}$, Na citrate $30 \mathrm{mM}$ ) and in PBS, blocked $10 \mathrm{~min}$ with $\mathrm{BSA} 2 \%$ in PBS. After a 30 min. incubation with a peroxydaseconjugated avidin-biotin complex (ABC kit, Vector) and subsequent wash with fresh buffer, labelling was monitored by a 2 min-reaction with $0.05 \% \mathrm{DAB}$ (diaminobenzidine, Sigma), $0.03 \% \mathrm{NiCl}_{2}, 0.6 \% \mathrm{H}_{2} \mathrm{O}_{2}$ in $50 \mathrm{mM}$ Tris- $\mathrm{HCl} \mathrm{pH} \mathrm{7.6.}$

\section{Serine protease activity assay}

Frozen olfactory organs or 13.S.1.24 cells were homogenized in the 'caspase lysis buffer' (ApoAlert caspase-3 assay, Clontech). Chymotrypsin-like activity assay was then performed by incubating $10 \mu \mathrm{g}$ of lysates in the presence of $10 \mu \mathrm{M}$ Suc-Ala-Ala-ProPhe-AMC fluorescent substrate in $0.1 \mathrm{M}$ Tris- $\mathrm{HCl} \mathrm{pH} 7,4 ; 10 \mathrm{mM} \mathrm{CaCl} 2$ reaction buffer. Fluorescence was measured after $3 \mathrm{~h}$ incubation at room temperature using the reader plate Victor (Wallac) with $380 \mathrm{~nm}$ excitation and $460 \mathrm{~nm}$ emission filters.

\section{Acknowledgements}

We thank Laure Granger, Nathalie Forey and Catherine Guix for excellent technical assistance. We wish to thank A LeCam for providing the Spi2 cDNA construct. L Pays was supported by a Ligue Nationale Contre le Cancer fellowship. This work was supported by the CNRS, the Ligue Nationale Contre le Cancer, the FRM, the ARC (9036,9340), the 'emergence' program.

\section{References}

1. Kuan CY, Roth KA, Flavell RA and Rakic P (2000) Mechanisms of programmed cell death in the developing brain. Trends Neurosci. 23: 291-297

2. Michel D, Moyse E, Brun G and Jourdan F (1994) Induction of apoptosis in mouse (correction of rat) olfactory neuroepithelium by synaptic target ablation. Neuroreport. 5: $1329-1332$
3. Calof AL, Hagiwara N, Holcomb JD, Mumm JS and Shou J (1996) Neurogenesis and cell death in olfactory epithelium. J. Neurobiol. 30: 67-81

4. Farbman Al, Buchholz JA, Suzuki Y, Coines A and Speert D (1999) A molecular basis of cell death in olfactory epithelium. J. Comp. Neurol. 414: 306-314

5. Cowan CM, Thai J, Krajewski S, Reed JC, Nicholson DW, Kaufmann SH and Roskams AJ. (2001) Caspases 3 and 9 send a pro-apoptotic signal from synapse to cell body in olfactory receptor neurons. J. Neurosci. 21: 7099-7109

6. Mahalik TJ (1996) Apparent apoptotic cell death in the olfactory epithelium of adult rodents: death occurs at different developmental stages. J. Comp. Neurol. 372: $457-464$

7. Crews L and Hunter D (1994) Neurogenesis in the olfactory epithelium. Perspect Dev. Neurobiol. 2: 151-161

8. Schwartz Levey M, Chikaraishi DM and Kauer JS (1991) Characterization of potential precursor populations in the mouse olfactory epithelium using immunocytochemistry and autoradiography. J. Neurosci. 11: 3556-3564

9. Deckner ML, Risling M and Frisen J (1997) Apoptotic death of olfactory sensory neurons in the adult rat. Exp. Neurol. 143: 132-140.

10. Michel D, Moyse E, Trembleau A, Jourdan F and Brun G (1997) Clusterin/ApoJ expression is associated with neuronal apoptosis in the olfactory mucosa of the adult mouse. J. Cell Science 110: 1635-1645

11. Whisstock J, Skinner R and Lesk AM (1998) An atlas of serpin conformations. Trends Biochem Sci. 23: 63-67.

12. Silverman GA et al (2001) The serpins are an expanding superfamily of structurally similar but functionally diverse proteins. Evolution, mechanism of inhibition, novel functions, and a revised nomenclature. J. Biol. Chem. 276: $33293-33296$

13. Potempa J, Korzus E and Travis J (1994) The serpin superfamily of proteinase inhibitors: structure, function, and regulation. J. Biol. Chem. 269: 15957-15960

14. Bird PI (1998) Serpins and regulation of cell death. Results Probl. Cell Differ. 24: 63-89

15. Inglis JD, Lee M, Davidson DR and Hill RE (1993) Isolation of two cDNAs encoding novel alpha 1-antichymotrypsin-like proteins in a murine chondrocytic cell line. Gene 106: 213-220

16. Coronas V, Feron F, Hen R, Sicard G, Jourdan F and Moyse E (1997) In vitro induction of apoptosis or differentiation by dopamine in an immortalized olfactory neuronal cell line. J. Neurochem. 69: 1870-1881

17. Llambi $F$, Causeret F, Bloch-Gallego $E$ and Mehlen $P$ (2001) Netrin-1 acts as a survival factor via its receptors UNC5H and DCC. EMBO J. 20: 2715-2722

18. Mehlen $P$, Rabizadeh S, Snipas SJ, Assa-Munt N, Salvesen GS and Bredesen DE (1998) The DCC gene product induces apoptosis by a mechanism requiring receptor proteolysis. Nature 395: 801-804

19. Yanagida M, Fukamachi H, Takei M, Uzumaki H, Saito TT, likura Yand Nakahata $T$ (1997) Effect of a chymotrypsin-like inhibitor, TPCK, on histamine release from cultured human mast cells. J. Pharm. Pharmacol. 49: 537-541

20. Lin Y, Devin A, Rodriguez Y and Liu ZG (1999) Cleavage of the death domain kinase RIP by caspase-8 prompts TNF-induced apoptosis. Genes Dev. 13: $2514-2526$

21. Houenou LJ, Turner PL, Li L, Oppenheim RW and Festoff BW (1995) A serine protease inhibitor, protease nexin I, rescues motoneurons from naturally occurring and axotomy-induced cell death. Proc. Natl. Acad. Sci. USA 9: 895899

22. Hsu PE, Yu F, Féron F, Pickes JO, Sneesby K, Mackay-Sim A (2001) Basic fibroblast growth factor and fibroblast growth factor receptors in adult olfactory epithelium. Brain Res. 896, 188-197

23. Doucette JR, Kiernan JA and Flumerfelt BA (1983) The re-innervation of olfactory glomeruli following transection of primary olfactory axons in the central or peripheral nervous system. J. Anat. 137:1-19

24. Doucette JR (1990) Glial influences on axonal growth in the primary olfactory system. Glia 3: 433-449

25. Roman-Cueto $A$ and Valverde $F$ (1995) Olfactory bulb ensheathing glia: a unique cell type with axonal growth-promoting properties. Glia. 14: 163-173

26. Schwarting GA, Kostek C, Ahmad N, Dibble C, Pays L and Puschel AW (2000) Semaphorin $3 A$ is required for guidance of olfactory axons in mice. J. Neurosci. 20: $7691-7697$

27. Li Y, Field PM and Raisman G (1997) Repair of adult rat corticospinal tract by transplants of olfactory ensheathing cells. Science 277: 2000-2002 
28. Zhou T, Song L, Yang P, Wang Z, Lui D and Jope RS (1999) Bisindolylmaleimide VIII facilitates Fas-mediated apoptosis and inhibits T cell-mediated autoimmune diseases. Nat. Med. 5: 42-48

29. Movsesyan VA, Yakovlev AG, Fan L and Faden Al (2001) Effect of serine protease inhibitors on posttraumatic brain injury and neuronal apoptosis. Exp. Neurology 167: $366-375$
30. Sandberg R, Yasuda R, Pankratz DG, CarterTA, Del Rio JA, Wodicka L, Mayford M, Lockhart DJ and Barlow C (2000) Regional and strain-specific gene expression mapping in the adult mouse brain. Proc. Natl. Acad. Sci. USA 97: $11038-11043$ 